\title{
SAR Reduction Using Integration of PIFA and AMC Structure for Pentaband Mobile Terminals
}

\author{
Jae-Gon Lee ${ }^{1}$ and Jeong-Hae Lee ${ }^{2}$ \\ ${ }^{1}$ Metamaterial Electronic Device Research Center, Hongik University, Seoul, Republic of Korea \\ ${ }^{2}$ Department of Electronic Information and Communication Engineering, Hongik University, Seoul, Republic of Korea
}

Correspondence should be addressed to Jeong-Hae Lee; jeonglee@hongik.ac.kr

Received 10 September 2016; Revised 6 December 2016; Accepted 4 January 2017; Published 31 January 2017

Academic Editor: Xianming Qing

Copyright ( 2017 Jae-Gon Lee and Jeong-Hae Lee. This is an open access article distributed under the Creative Commons Attribution License, which permits unrestricted use, distribution, and reproduction in any medium, provided the original work is properly cited.

\begin{abstract}
In this paper, a capacitive grating artificial magnetic conductor (AMC) is presented to reduce the specific absorption rate (SAR) in pentaband mobile terminals. The AMC structure is implemented using a dielectric film with the printed arrays of the metal strips placed at the top and the bottom of the dielectric. It is difficult to design the AMC structure to operate at low $(824 \sim 960 \mathrm{MHz})$ and high bands (1710 2170 MHz) simultaneously, because of the limited space available for the antenna. Hence, we have designed the capacitive grating AMC to operate at a high band. Then, we attached a PIFA to the AMC structure to cover low and high bands. As the AMC structure is operated as a perfect electric conductor (PEC) in low band, the radiating branches of the PIFA for the low and high bands should be located on the non-AMC and the AMC structures, respectively. Even though the AMC structure is operated at a high band, the effect against the head could be reduced in the pentaband due to the spreading effect of the electromagnetic (EM) field at lower bands. From measured results, the $1 \mathrm{~g}$ SAR in the case of the AMC antenna is significantly lower than that in the case where only the PIFA is present in the pentaband.
\end{abstract}

\section{Introduction}

The specific absorption rate (SAR) is a measure of the rate at which energy is absorbed by the human body when exposed to a radio frequency (RF) electromagnetic (EM) field. It is defined as the power absorbed per mass of tissue and is expressed in units of watts per kilogram $(\mathrm{W} / \mathrm{kg})$. For mobile terminals, the recommended localized head and body SAR limit is $2 \mathrm{~W} / \mathrm{kg}$ in a $10 \mathrm{~g}$ average mass in the shape of a cube. Some countries such as the Republic of Korea (ROK), the US, and Canada have adopted slightly different SAR limits of $1.6 \mathrm{~W} / \mathrm{kg}$ in a $1 \mathrm{~g}$ average mass in the shape of a cube [1]. In general, most mobile terminal engineers struggle to meet the standard for head SAR at high bands because of the increase in electrical conductivity with frequency. When measuring the SAR value in the case of mobile terminals, the phone is held close to the head in the talk position. The SAR value is then measured at the location that has the highest absorption rate on the entire head area. Although the main antenna is generally at the bottom of the phone, the SAR values frequently failed to satisfy the SAR standard. Furthermore, long term evolution advanced (LTE-A) offers considerably higher data rates than the initial releases of LTE. While the spectrum usage efficiency has been improved, this alone cannot provide the data rates that are required from LTE-A. To achieve these high data rates, it is necessary to increase the transmission bandwidths beyond those that can be supported by a single carrier. The method being proposed is termed carrier aggregation (CA). LTE-A CA makes it possible to utilize more than one carrier and, thereby, increase the overall transmission bandwidth. In addition, most mobile carriers plan to provide higher data rates at up-link and mobile terminals require $2 \mathrm{Tx}$ as well as $2 \mathrm{Rx}$ antennas. As the secondary antenna is generally positioned at the top of the mobile terminal, the additional Tx antenna is very close to the head. This may result in issues such as a high SAR value.

Therefore, we need a fundamental solution that can ensure high efficiency and a low SAR value and which can be developed quickly and cost-effectively. The artificial magnetic conductor (AMC) [2] can be the most suitable of 


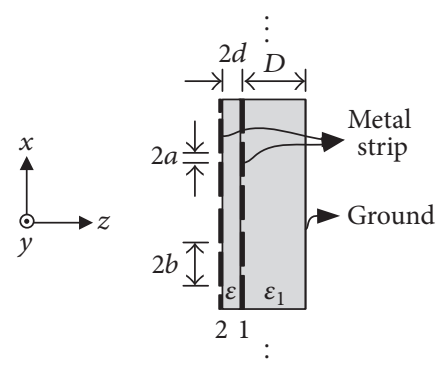

(a)

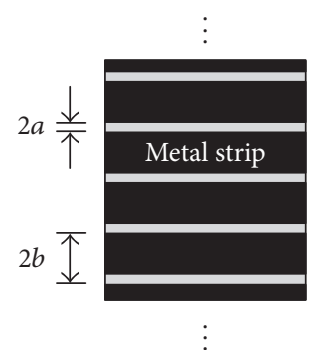

(b)

FIGURE 1: Capacitive grating AMC structure: (a) side view; (b) top view.

all candidates in designing a low-profile antenna with a low SAR value because the AMC has a zero-degree reflection phase. Previous studies [3-8] have proposed using the AMC structure for SAR reduction. The proposed AMC structures were bulky and could not be placed in multiband mobile terminals, which made them unsuitable for use in mobile handsets. Hence, a capacitive grating AMC has been designed to reduce the SAR in pentaband mobile terminals (GSM 850/ 900/1800/1900/W2100 bands, 824 894 MHz/880 960 MHz/ $1710 \sim 1880 \mathrm{MHz} / 1850 \sim 1990 \mathrm{MHz} / 1920 \sim 2170 \mathrm{MHz}$ ), which is presented in this paper. As the available space for the antenna in a mobile terminal is very small, we have designed the AMC structure to operate at a high band (GSM 1800/1900/W2100 bands). Then, a planar inverted-F antenna (PIFA) was designed to cover the pentaband. The radiating branches of the PIFA for the low and high bands were placed in the nonAMC and AMC structures, respectively. The implementation method of the integrated antenna with the AMC structure is described and the measured total radiated power (TRP) and $1 \mathrm{~g}$ SAR values between PIFA with and without the AMC structure in the pentabands are compared.

\section{Design of AMC Structure and PIFA}

In order to reduce the SAR values, we have designed and used a capacitive grating AMC structure [9]. Figures 1 and 2 show the capacitive grating AMC structure with double layers and its equivalent transmission line, respectively. The dielectric film used here has the printed arrays of the metal strips placed at the top and the bottom of the dielectric. When the incident plane wave is perpendicular to the AMC structure, the impedance $(Z)$ between the first and the second conductive layers can be expressed as (1). Impedance $\left(Z_{11}\right)$ between the second conductive layer and the conductive ground layer can be expressed as (2). Therefore, the total impedance $\left(Z_{22}\right)$ can be obtained from (3) [9]:

$$
\begin{aligned}
Z & =\frac{-j d \lambda}{\pi \varepsilon(b-2 a) b} \\
Z_{11} & =j k D \quad\left(k \sqrt{\varepsilon_{1}} D \leq 1\right) \\
Z_{22} & =\frac{Z_{11} Z}{Z_{11}+Z}
\end{aligned}
$$

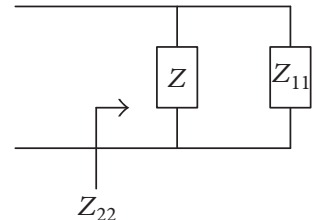

Figure 2: Equivalent transmission line of the AMC structure.

where $\varepsilon$ and $\varepsilon_{1}$ are the permittivity of the material placed between the gratings and the layer located between the pair of gratings and the ground plane, respectively. The dimensions of $a, b, d$, and $D$ are shown in Figure 1 . In order to operate a high impedance surface (HIS), $Z_{22}$ must be an infinite value and the denominator of $Z_{22}$ must be equal to zero. Therefore, the operating frequency for HIS can be calculated by

$$
\text { Freq }_{\text {HIS }}=\frac{c}{\pi \sqrt{2} \sqrt{\varepsilon D b(b-2 a) / d}},
$$

where $c$ is the velocity of light. The dimensions of the designed AMC structure are as follows: $\varepsilon=4.4, \varepsilon_{1}=1, a=0.1 \mathrm{~mm}$, $b=4 \mathrm{~mm}, d=0.2 \mathrm{~mm}$, and $D=4 \mathrm{~mm}$. From (4), we can expect that the analytic center frequency operated to high impedance surface $\left(\right.$ Freq $_{\mathrm{HIS}}$ ) is $1846 \mathrm{MHz}$. Further, using the full wave simulator (ANSYS HFSS), we can calculate the reflection phase of the designed AMC structure. We have set up an infinite periodic structure using the master and slave boundary condition and a normally incident plane wave with Ex polarization. The AMC range is generally defined from $-90^{\circ}$ to $90^{\circ}$ for the reflection phase and is simulated from $1690 \mathrm{MHz}$ to $2180 \mathrm{MHz}$, as shown in Figure 3. Freq $_{\text {HIs }}$ can be controlled using the parameters of the AMC structure such as height of the lower substrate $(D)$, height of the upper substrate $(2 d)$, and periodicity $(b)$. Among the parameters, the dominant factors are the height of the upper substrate $(2 d)$ and the height of the lower substrate $(D)$. If the height of the upper substrate $(2 d)$ and that of the lower substrate $(D)$ decrease and increase, respectively, Freq ${ }_{\mathrm{HIS}}$ can be downshifted.

As the capacitive grating AMC structure with double layers in Figure 1(a) is a model of an infinite structure, we need a finite capacitive grating AMC structure to employ in mobile handsets. The finite AMC structure should have metalized side connected to the main board to prevent wave leaked to 


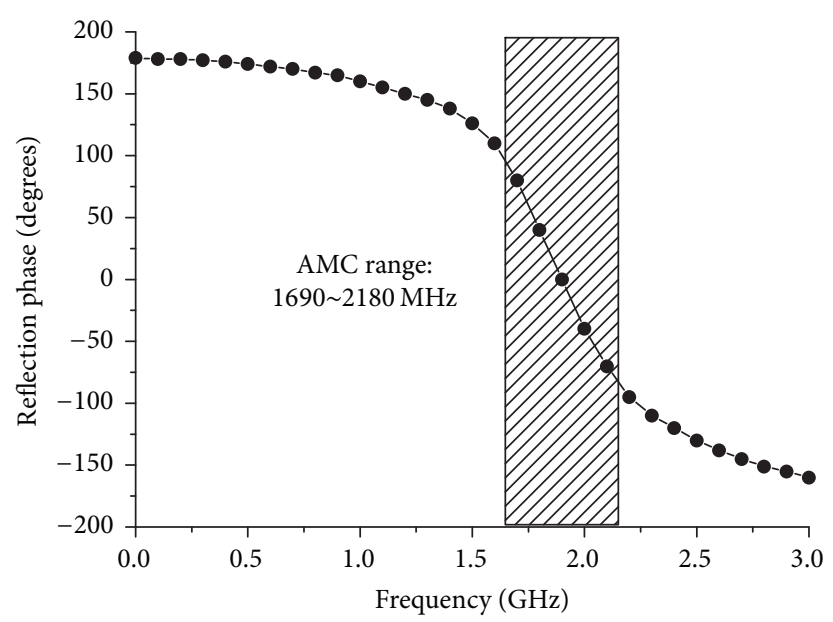

FIGURE 3: Full wave simulated reflection phase of the capacitive grating AMC structure.

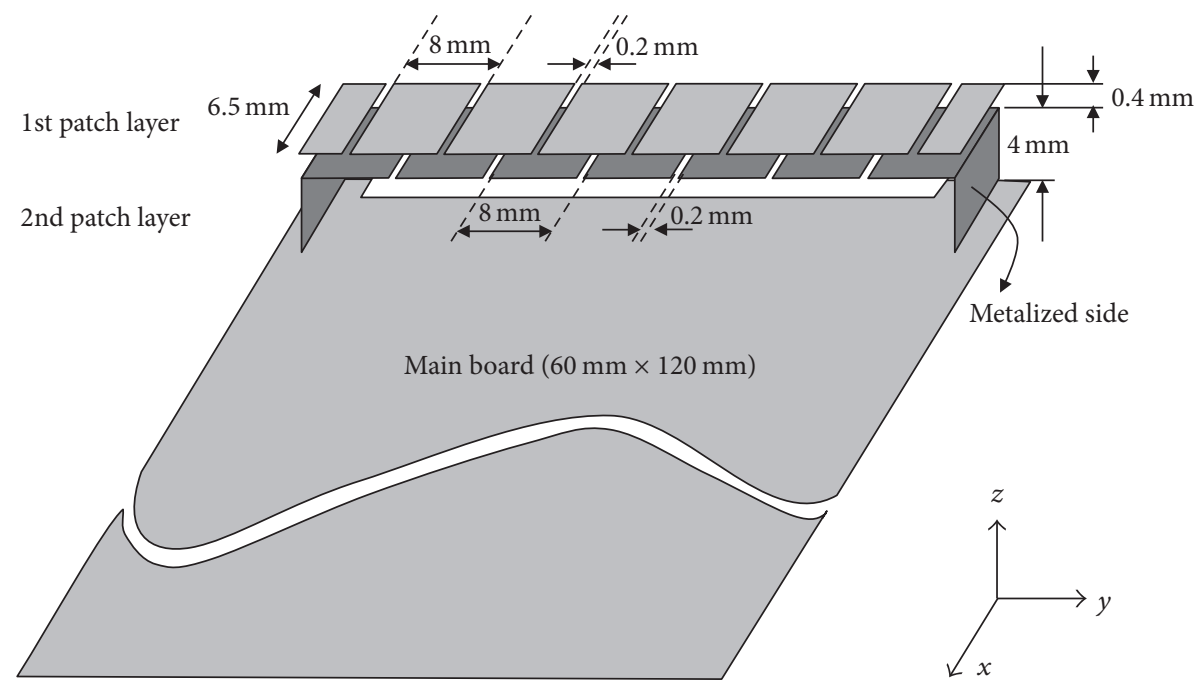

FIGURE 4: Designed capacitive grating AMC structure for implementation in mobile terminal.

the side direction, as shown in Figure 4 [10]. Additionally, the ground plane beneath the AMC structure should be removed by $2 \mathrm{~mm}$ to increase the efficiency and bandwidth of PIFA at low bands. The overall dimensions of the antenna including the AMC structure are $60 \mathrm{~mm}$ (length) $\times 10 \mathrm{~mm}$ (width) $\times$ $5 \mathrm{~mm}$ (height). If the width of the AMC structure increases, it results in a greater SAR reduction but the efficiency of the antenna is degraded at a low band. After optimization, the width of the AMC structure and that of the non-AMC structure are designed to be $6.5 \mathrm{~mm}$ and $3.5 \mathrm{~mm}$, respectively.

In this paper, the PIFA and the capacitive grating AMC structure operate at both low and high bands and at only high band, respectively. This is because the available antenna space in the mobile terminal is not sufficient to design an AMC structure that can be operated at both low and high bands. Therefore, we need a novel technique that uses an integrated PIFA and AMC structure. As the AMC structure operates like a perfect electric conductor (PEC) at a low band, the radiating branches of the PIFA for the low and high bands should be located on the non-AMC and the AMC structures, respectively, as shown in Figure 5. At a high band, the current distribution in the AMC structure and in the PIFA is in the same direction. This makes it possible to mount the PIFA close to the AMC structure unlike in the case of an ordinary metal surface. The dimensions of the PIFA are determined simultaneously with the parameters of the grounded AMC structure. To obtain broad and multiimpedance matching conditions for the pentaband (824 $960 \mathrm{MHz}$ and 1710 2170 MHz), we have optimized the length and shape of the PIFA on the AMC structure. The lengths of the antenna patterns for the low and high band are $65 \mathrm{~mm}$ and $30 \mathrm{~mm}$, respectively.

Also, a material and a thickness of supporter between the AMC structure and the antenna pattern are Teflon with a permittivity of 2.1 and $0.5 \mathrm{~mm}$, respectively. The grounded AMC structure on the telephone chassis and the PIFA form a low-profile and low-complexity mobile phone terminal. The integrated antenna has the properties of higher efficiency and 


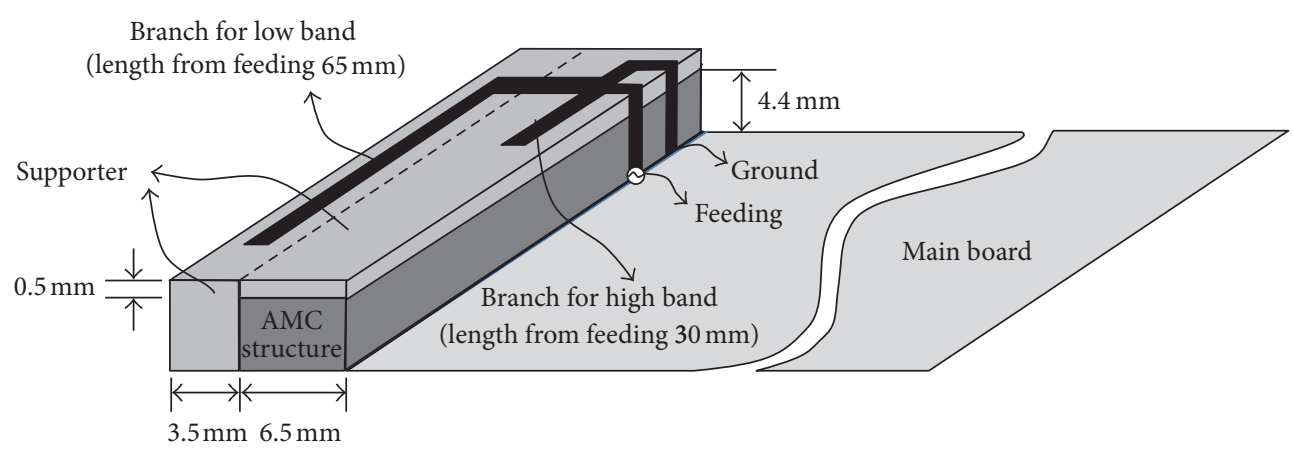

(a)

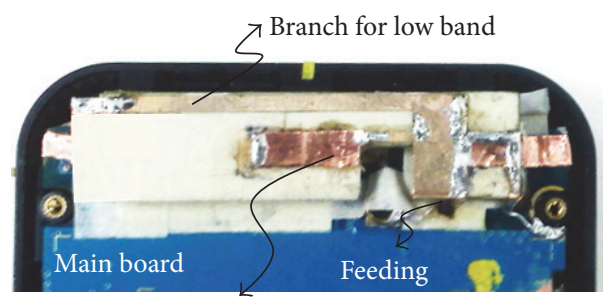

Branch for high band

(b)

FIGURE 5: (a) Designed integrated antenna composed of PIFA and AMC structure. (b) Photograph of the proposed antenna.

lower SAR values for a pentaband when compared with a conventional PIFA without an AMC structure.

\section{Measured Results and Discussion}

We have measured and compared the performances of a pentaband antenna with and without an AMC structure, where the antenna is located at the bottom of the phone using an Agilent 8510C vector network analyzer. As shown in Figure 6 , the voltage standing wave ratio (VSWR) of the PIFA on the AMC structure is compared with that of PIFA without AMC structure. It can be confirmed that even though the PIFA is integrated with the AMC structure, the impedance matching is nearly the same as that of the PIFA without the AMC structure.

Moreover, the measured VSWR of the proposed antenna is in good agreement with the simulated result except for the shifted resonance frequency at high bands. We have measured the total radiated power (TRP) with and without a head phantom in an anechoic chamber. The input powers of the GSM850, GSM900, GSM1800, GSM1900, and W2100 bands are $33 \mathrm{dBm}, 33 \mathrm{dBm}, 30 \mathrm{dBm}, 30 \mathrm{dBm}$, and $23 \mathrm{dBm}$, respectively. The total efficiencies of the PIFA on the AMC structure with a head phantom are measured to be $13 \%$, 9\%, 19\%, 24\%, and 28\% in GSM850, GSM900, GSM1800, GSM1900, and W2100 bands, respectively. Figure 7 shows the measured far-field radiation pattern $(x-z$ plane $)$ at $850 \mathrm{MHz}$ and $1900 \mathrm{MHz}$. The radiation in the direction of the head in the proposed antenna is reduced at both low and high bands when compared with PIFA without an AMC structure. In other words, the radiation pattern towards the head is

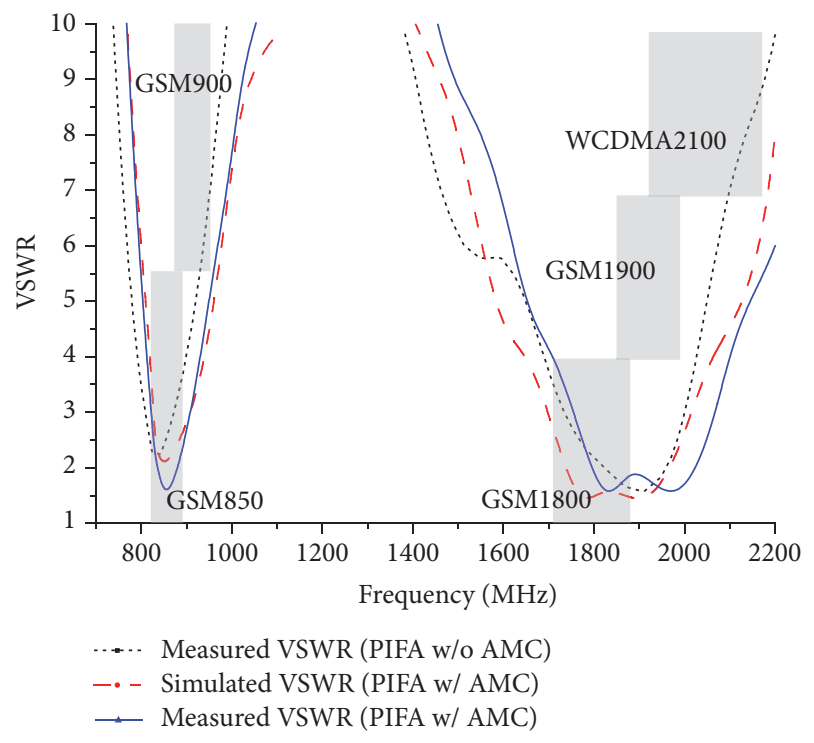

FIgURE 6: Comparison of VSWR between PIFA with and without the AMC structure.

blocked by the AMC structure without reduction of radiation efficiency. In order to design an antenna on the PEC that reduces the SAR value without reduction of radiation efficiency, the distance between the antenna and the PEC should be a quarter wavelength. Even if the TRP in both cases is nearly the same without a head phantom, the TRP of the proposed antenna with a head phantom is equal to or higher than that of PIFA without an AMC structure, as shown in 


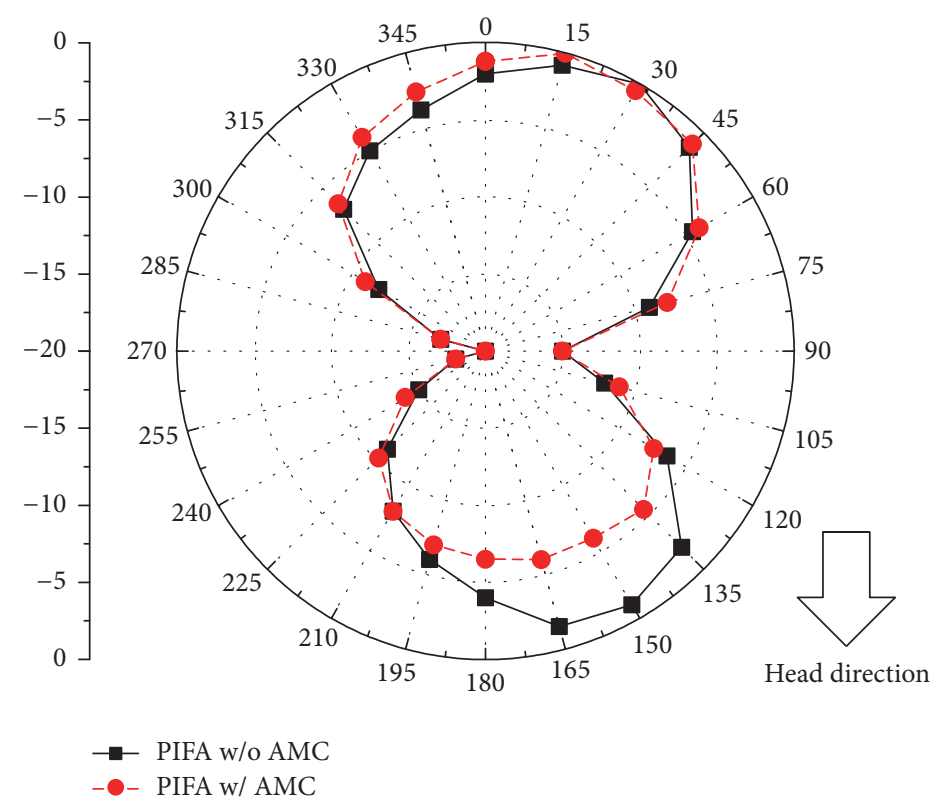

(a)

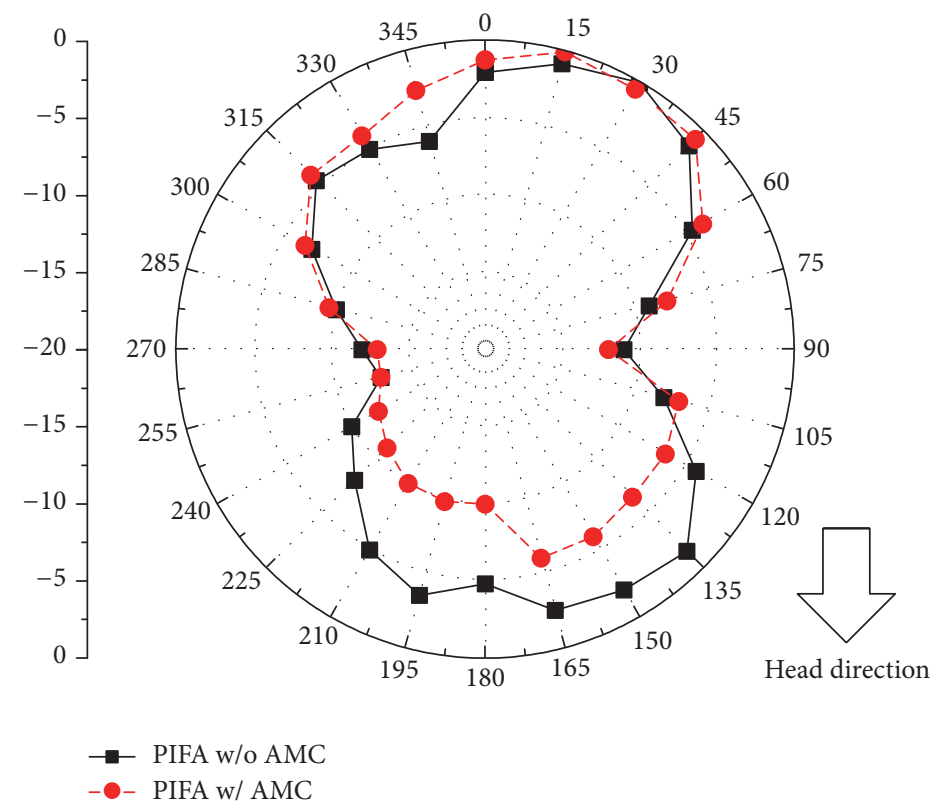

(b)

FIGURE 7: Comparison of measured far-field radiation patterns between PIFA with and without the AMC structure ( $x$ - $z$ plane). (a) Low band (850 MHz). (b) High band (1900 MHz).

Figure 8(a). The reason is that the AMC structure decreases the effect of the PIFA against the head. For the same reasons, $1 \mathrm{~g}$ SAR in the case of the integrated AMC antenna decreases compared with an antenna without the AMC structure. In the case of the proposed antenna, the $1 \mathrm{~g}$ SAR values were measured to be $0.9 \mathrm{~W} / \mathrm{kg}, 0.7 \mathrm{~W} / \mathrm{kg}, 0.5 \mathrm{~W} / \mathrm{kg}, 0.5 \mathrm{~W} / \mathrm{kg}$, and $0.8 \mathrm{~W} / \mathrm{kg}$ in GSM850, GSM900, GSM1800, GSM1900, and W2100 band, respectively, using Speag's DASY4 SAR measurement system. The DASY4 consists of a robotic arm with an $E$-field probe, a robot controller, a measurement server, a phantom with a tissue-equivalent liquid, and a device holder as shown in Figure 9. This means that the $1 \mathrm{~g}$ SAR has decreased by $25 \%, 36.4 \%, 37.5 \%, 28.5 \%$, and $27 \%$ for each band (GSM850, GSM900, GSM1800, GSM1900, and W2100 bands) in the proposed antenna when compared with PIFA without the AMC structure. Despite the fact that the head TRP of the proposed antenna is higher than that of PIFA without the AMC structure, the SAR values in the high bands are substantially reduced. Particularly, the $1 \mathrm{~g}$ SAR at low bands has also reduced despite the AMC structure operating in the high band. It seems that the AMC structure has the characteristic of a metal at low bands, so that the EM field of 


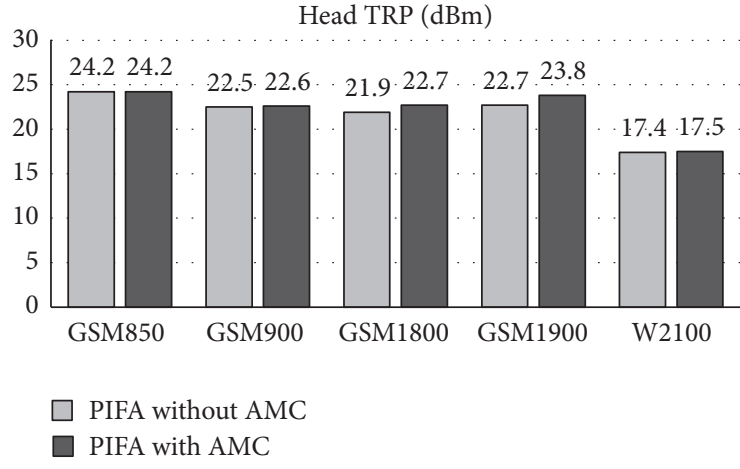

(a)

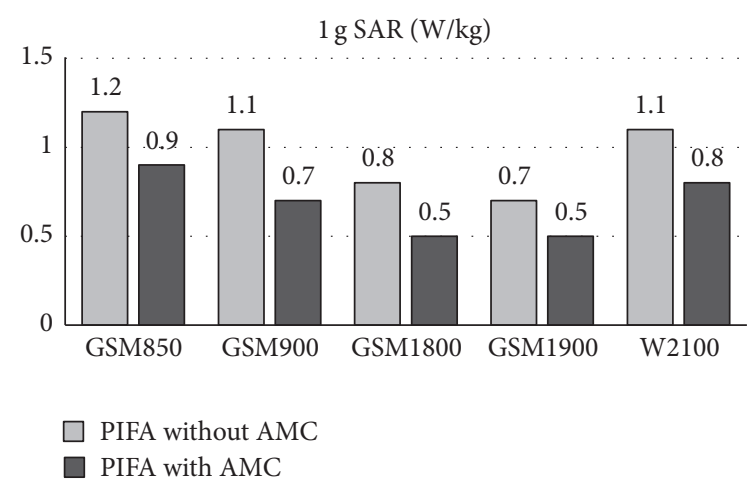

(b)

FIGURE 8: Comparison of head TRP and $1 \mathrm{~g}$ SAR between PIFA with and without the AMC structure (antenna position: bottom of phone). (a) Head TRP. (b) $1 \mathrm{~g}$ SAR.

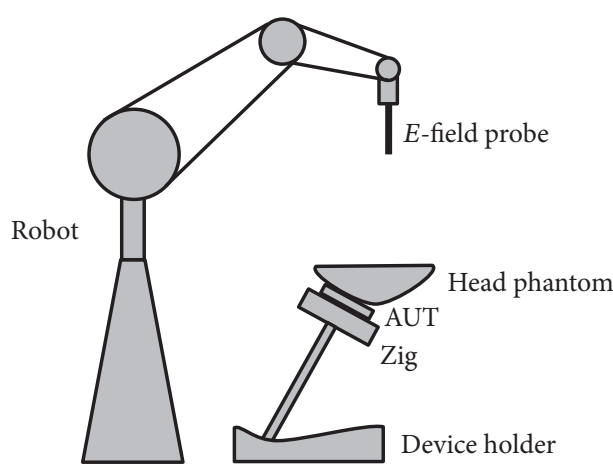

(a)

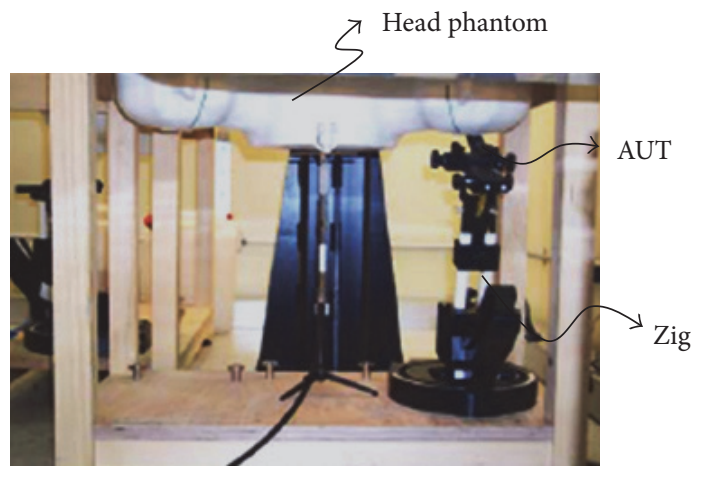

(b)

Figure 9: SAR measurement system (Speag's DASY 4). (a) Layout. (b) Photograph of the system.

the end point of the antenna spreads to the AMC structure due to change in the ground condition.

\section{Conclusion}

In this paper, a capacitive grating AMC structure is presented and designed to reduce the SAR value, and the integrated antenna implemented with a PIFA on the AMC structure is optimized for covering the pentaband. As the AMC structure operates at low and high bands simultaneously, it cannot be inserted in the mobile terminal. Hence, we have designed the AMC structure to operate at high bands alone and have placed branches of the PIFA on the non-AMC and the AMC structures to operate it at low and high bands, respectively. The AMC structure is capable of blocking the radiation pattern towards the head without reducing the antenna efficiency because the reflection wave of the antenna close to the AMC structure generates constructive rather than destructive wave. As a result, the $1 \mathrm{~g}$ SAR decreases by $25 \%$, $36.4 \%, 37.5 \%$, 28.5\%, and 27\% for the GSM850, GSM900, GSM1800, GSM1900, and W2100 bands, respectively, when compared with that of the PIFA without the AMC structure. Due to the spreading effect of the EM field in the low band, we achieved SAR reduction in the pentaband. Therefore, we have found a fundamental solution for SAR reduction that does not degrade the head TRP in the pentaband.

\section{Competing Interests}

The authors declare that there is no conflict of interests regarding the publication of this paper.

\section{Acknowledgments}

This research was supported by Basic Science Research Program through the National Research Foundation of Korea (NRF) funded by the Ministry of Education (no. 2015R1A6A1A03031833).

\section{References}

[1] IEEE Standards, "IEEE standard for safety levels with respect to exposure to radio frequency electromagnetic fields, $3 \mathrm{kHz}$ to 300 GHz," IEEE Standard C95, 2005.

[2] D. Sievenpiper, L. Zhang, R. F. Jimenez Broas, N. G. Alexöpolous, and E. Yablonovitch, "High-impedance electromagnetic surfaces with a forbidden frequency band," IEEE Transactions on Microwave Theory and Techniques, vol. 47, no. 11, pp. 20592074, 1999. 
[3] J.-N. Hwang and F.-C. Chen, "Reduction of the peak SAR in the human head with metamaterials," IEEE Transactions on Antennas and Propagation, vol. 54, no. 12, pp. 3763-3770, 2006.

[4] M. R. I. Faruque, N. Misran, and M. T. Islam, "Split ring resonators for SAR reduction in human head," in Proceedings of the 17th Asia Pacific Conference on Communications (APCC '11), pp. 880-883, October 2011.

[5] S. I. Kwak, D.-U. Sim, and J. H. Kwon, "Design of optimized multilayer PIFA with the EBG structure for SAR reduction in mobile applications," IEEE Transactions on Electromagnetic Compatibility, vol. 53, no. 2, pp. 325-331, 2011.

[6] S. I. Kwak, D. U. Sim, J. H. Kwon, and H. D. Choi, "Experimental tests of SAR reduction on mobile phone using EBG structures," Electronics Letters, vol. 44, no. 9, pp. 568-570, 2008.

[7] R. Ikeuchi and A. Hirata, "Dipole antenna above EBG substrate for local SAR reduction," IEEE Antennas and Wireless Propagation Letters, vol. 10, pp. 904-906, 2011.

[8] K. Han, M. Swaminathan, R. Pulugurtha et al., "Magnetodielectric nanocomposite for antenna miniaturization and SAR reduction," IEEE Antennas and Wireless Propagation Letters, vol. 15, pp. 72-75, 2016.

[9] Y. N. Kazantsev and V. N. Apletalin, "Artificial magnetic conductors based on capacitive gratings," Journal of Communications Technology and Electronics, vol. 52, no. 4, pp. 390-398, 2007.

[10] J.-G. Lee and J.-H. Lee, "SAR reduction using integration of PIFA and AMC structure for penta-band mobile terminals," in Proceedings of the Korea Electromagnetic Engineering Society Summer General Conference, p. 137, 2016. 


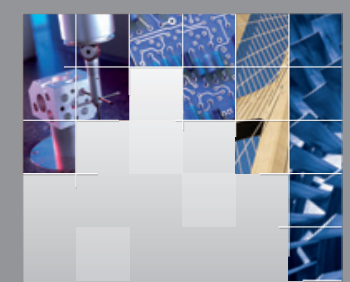

\section{Enfincering}
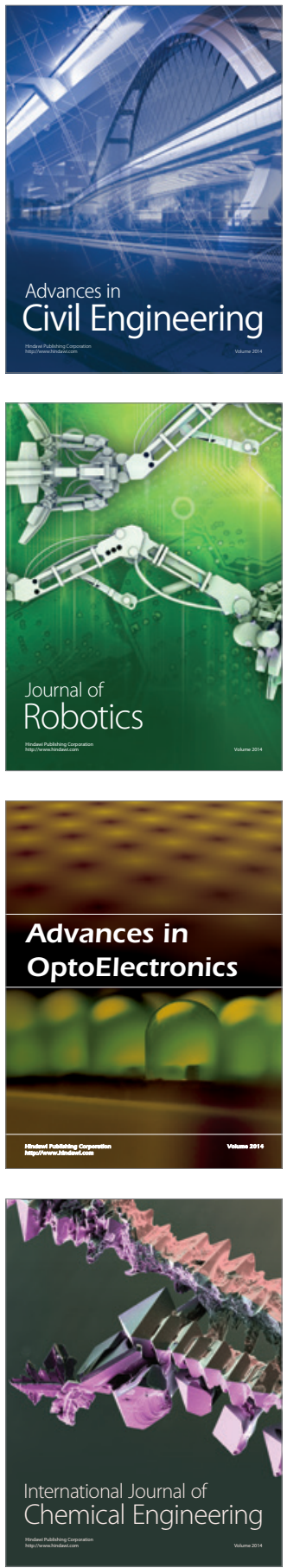

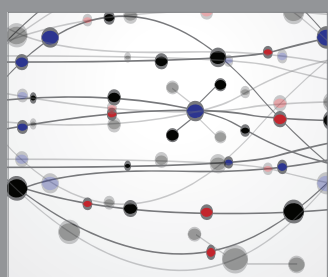

The Scientific World Journal

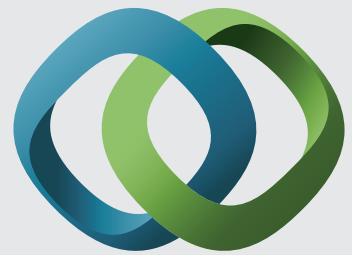

\section{Hindawi}

Submit your manuscripts at

https://www.hindawi.com
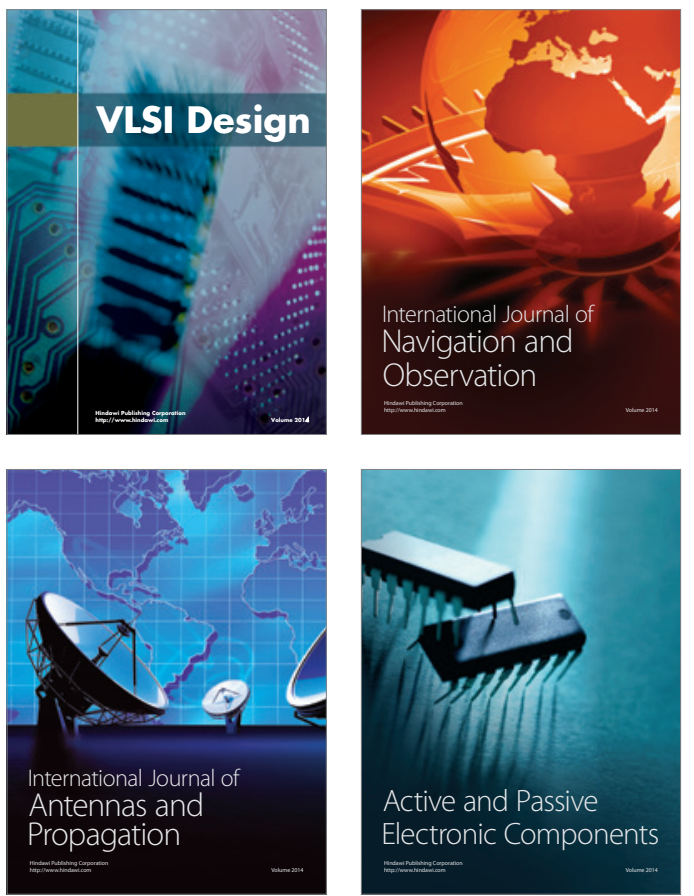
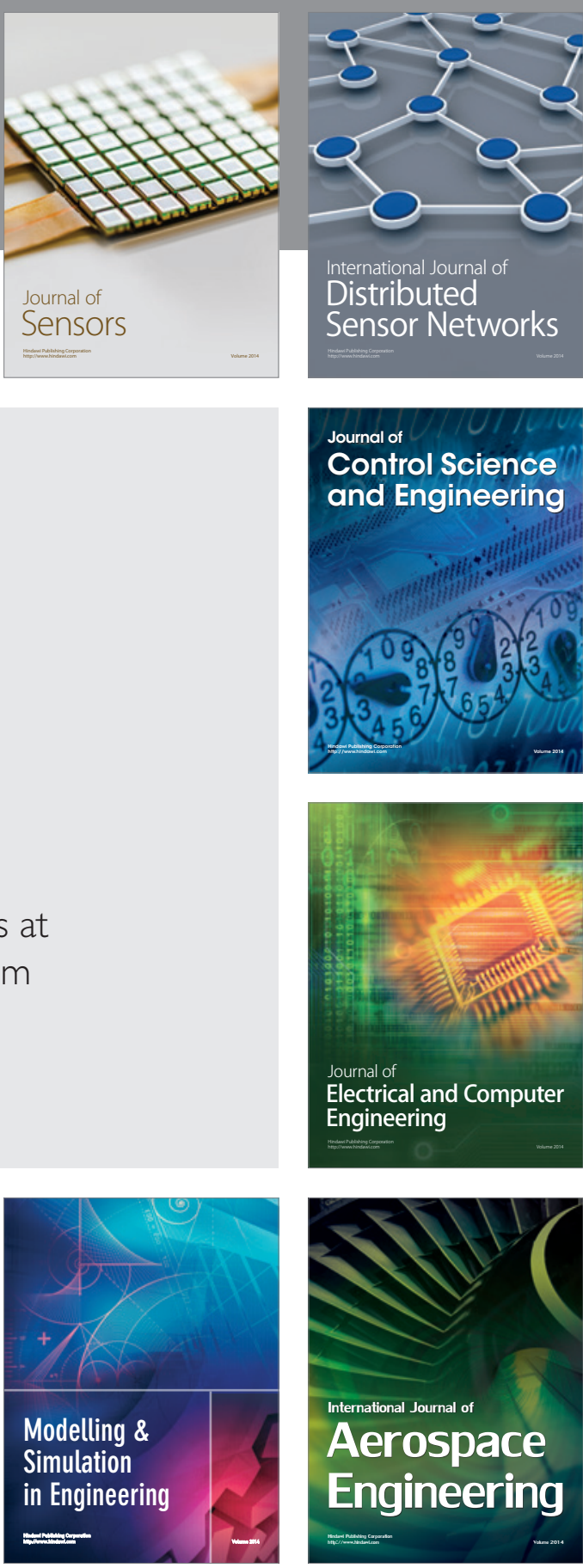

International Journal of

Distributed

Sensor Networks

$-$

Joumal of

Control Science

and Engineering
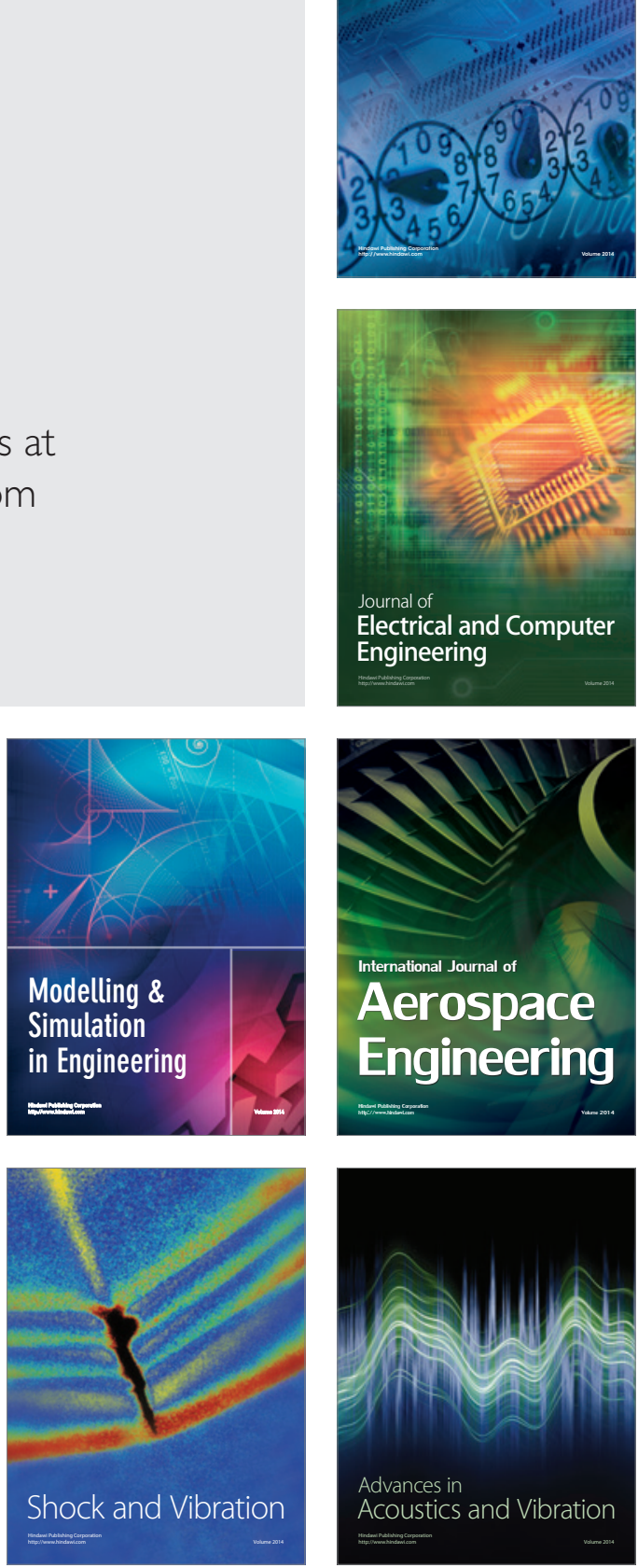